Modern Physics Letters A,

(C) World Scientific Publishing Company

\title{
AN EFFECTIVE QUANTUM MECHANISM FOR MASS GENERATION IN DIFFEOMORPHISM-INVARIANT THEORIES*
}

\author{
J. L. JARAMILLO \\ V. ALDAYA \\ Instituto Astrofísica Andalucía (CSIC), Apartado Postal 3004 \\ Granada 18080, Spain \\ Instituto Carlos I de Física Teórica y Computacional, Facultad de Ciencias, \\ Universidad de Granada, Campus de Fuentenueva \\ Granada 18002, Spain
}

\begin{abstract}
We propose a scenario for particle-mass generation, assuming the existence of a physical regime where, firstly, physical particles can be considered as point-like objects moving in a background space-time and, secondly, their mere presence spoils the invariance under the local diffeomorphism group, resulting in an anomalous realization of the latter. Under these hypotheses, we describe mass generation starting from the massless free theory. The mechanism is not sensitive to the detailed description of the underlying theory at higher energies, leaning only on general structural features of it, specifically diffeomorphism invariance.
\end{abstract}

The problem we address in this work is the origin of particle masses. Even though a strong emphasis has been placed on this issue throughout the development of modern physics, the subject seems far from being resolved.

The correction of the mass of a particle as an effective consequence of its interaction with the surrounding environment is a very old idea that can be tracked to nineteenth-century hydrodynamics. In fact, for many different physical systems describing the motion of an object inside a classical continuum fluid, the solution of hydrodynamical equations admits an effective treatment in which the object behaves as in free motion with a corrected or renormalized mass which depends on general features such as boundary conditions. The extension of these ideas to electrodynamics led J.J.Thomson to the introduction of the notion of electromagnetic mass of a charge as a consequence of the interaction with its own electromagnetic field, fundamental element in the later Lorentz's theory of the electron 1 . With the arrival of the Quantum Theory, the efforts by Kramers (largely inspired in Lorentz's insights) resulted in the connection between the previous classical ideas and the new

*Work partially supported by the DGICYT. 
problems related to the divergences appearing in the calculation of the electron selfenergy, leading to a radiative mass renormalization. In the early days of Quantum Electrodynamics there was hope of developing a fundamental theory that would eliminate the divergences and successfully derive the actual values of its characteristic parameters. However, the eventual resolution of the problem by implementing the renormalization program finally led to a situation in which Quantum Field Theory (QFT) appears as an effective theory. In fact, physics beyond a certain energy scale is not probed, though the renormalization of certain parameters of the model, among them the masses of the particles, non-trivially affects lower-energy physics. In this scenario, the idea of mass as self-energy has withered away to moot status. Nevertheless, conceptually different mechanisms can be devised for addressing partial yet fundamental aspects of the mass-origin problem, an example of which is the use of lattice QCD techniques for light hadrons 2. In any case, questions such as lepton masses or cosmological dark matter, remain open.

From this historical detour (see 1 for further details), we extract our two main guidelines. Firstly, we take up the old idea of emphasizing the interaction with the surrounding fields as fundamental in the generation of mass and, secondly, we adopt an effective aproach in which physics beyond a certain scale is not discussed. The presence of unanswered questions suggests the introduction of physics often ignored in the mass generation problem. An appealing (and obvious) candidate for the missing physical ingredient is Gravity with its associated diffeomorphism invariance, generally not considered in high-energy particle physics. Therefore, the only explicit condition we shall impose on the underlying fundamental theory is an essential role for the notion of diffeomorphism invariance.

When adopting the above-mentioned effective attitude, it seems reasonable to admit the existence of a scale of energies in which standard QFT is a good approximation, and its notion of a particle as a local excitation of the vacuum resulting from the action of a local field operator applies. We are also implicitly accepting a notion of space-time as a differentiable manifold making up the background in which particles move. We shall phenomenologically separate the intrinsic dynamics of this effective background, governed by classical General Relativity, from the effect that the underlying diffeomorphism invariance could exert in the quantum proccess of particle creation.

We are therefore studying a regime in which physical particles can be considered as point-like objects and the classical dynamics of space-time is decoupled (adiabatic condition). The adjective physical appearing here is essential, as opossed to the ideal test particles, causing an effective breakdown of the space-time notion at the point itself on which the particle lies. We are suggesting that physical particles literally pierce space-time, producing a hole. This has profound consequences in the quantum model describing particle creation. It can be shown 4 that the presence of a hole in a two-dimensional manifold induces anomalous (central) terms in the quantum commutators between (some of) the generators of diffeomorphism invariance, thus spoiling this classical symmetry (even though this can be properly healed in spe- 
cific theories). We propose that this phenomenon generalizes to realistic space-time dimensions, inducing an anomalous realization of classical diffeomorphism symmetry in the effective quantum process of particle creation, something that could be supported by the analysis of the leading terms of appropiate Operator Product Expansions. This does not contradict an exact implementation of this symmetry at higher energies, when using a more fundamental model for the coupling between the gravitational and matter degrees of freedom. It simply means that the price we must pay for avoiding such a detailed description, and admitting an effective treatment in which classical space-time is decoupled, is the acceptance of a breakdown of classical diffeomorphism gauge invariance.

The previous heuristic motivations can be synthesized in the following hypothesis: there exists an effective regime in which physical particles are point-like and their creation process entails a breakdown of classical diffeomorphism invariance, the latter being realized in an anomalous way.

The presence of an anomaly in a local gauge theory obstructs the reduction of degrees of freedom for which the gauge symmetry is devised, entailing an enlargement of the physical phase space performed by the spurious (in principle) modes ${ }^{a}$. This issue poses serious concerns for the consistency of the theory, at least when applying standard techniques, something especially critical when addressing the gauge theory as fundamental (attempts to construct consistent anomalous theories do exist 1 and the above-mentioned explicit appearance of extra degrees of freedom can be made apparent). However, the presence of an anomaly can also be interpreted as a signature for understanding the theory as a low-energy effective model, indicating the existence of new physics at higher energies 6 . This is precisely the situation we are dealing with here. The influence of higher-energy degrees of freedom is encoded in some effective degrees of freedom arising in the anomalous low-energy theory.

A standard way in which an anomaly manifests itself, in accordance with the considerations above, is through the appearance of extra terms in the quantum commutators with respect to the ones defining the classical symmetry. Therefore, we propose that the diffeomorphism symmetry is realized in the effective theory as an extension (not necessarily central) of the classical local diffeomorphism algebra. For concretepess we focus on the tensorial extensions, which are classified in 1 and discussed in 8 . We shall work in momentum space and denote the diffeomorphism generators by $\hat{L}_{\mu}(\mathbf{m})$, the fields corresponding to the particles generically by $\hat{\Phi}_{a}(\mathbf{m})$ and the tensorial extensions by $\hat{A}_{i}(\mathbf{m})$ ( $\mu$ is a space-time index, $a$ and $i$ internal indices and $\mathbf{m}$ a vector labelling momentum space). In this notation, the quantum brackets are given by:

$$
\begin{aligned}
& {\left[\hat{L}_{\mu}(\mathbf{m}), \hat{L}_{\nu}(\mathbf{n})\right]=m_{\nu} \hat{L}_{\mu}(\mathbf{m}+\mathbf{n})-n_{\mu} \hat{L}_{\nu}(\mathbf{m}+\mathbf{n})+c_{\mu \nu}^{i}(\mathbf{m}, \mathbf{n}) \hat{A}_{i}(\mathbf{m}+\mathbf{n})} \\
& {\left[\hat{L}_{\mu}(\mathbf{m}), \hat{\Phi}_{a}(\mathbf{n})\right]=-n_{\mu} \hat{\Phi}_{a}(\mathbf{m}+\mathbf{n})}
\end{aligned}
$$

a A familiar example of this phenomenon in string theory is the Liouville mode in the non-critical string. 


$$
\left[\hat{\Phi}_{a}(\mathbf{m}), \hat{\Phi}_{b}(\mathbf{n})\right]=\hat{\alpha}_{a b}(\mathbf{m}, \mathbf{n})
$$

where $c_{\mu \nu}^{i}(\mathbf{m}, \mathbf{n})$ is the cocycle linked to the anomalous extension giving effective dynamical content to the diffeomorphisms and $\hat{\alpha}_{a b}(\mathbf{m}, \mathbf{n})$ provides the standard commutators of the free fields.

To give a specific meaning to the entire foregoing discussions, we need to construct explicitly a physical model describing dynamics consistent with the algebra (11). A particularly well suited formalism for such a task is the so-called Group Approach to Quantization (GAQ, see $)$. In short, the main achievement of this approach is the construction of physical dynamics out of a given Lie algebraic structure taken as the only physical input. The technique, in some points, resembles Kirillov's construction of dynamics on the coadjoint orbits of a gropp 10 and shares some important general features with Geometric Quantization 11. The final outcome is an explicit unitary and irreducible representation of the operators in the starting Lie algebra.

When applying these techniques to algebras of the type (11), we obtain maximumweight representations (possessing a unique vacuum in the Hilbert space), where the corresponding diffeomorphism operators $\hat{L}_{\mu}(\mathbf{m})$ act and genuinely raise and lower the physical states, according to their gained dynamical content. A most important precise and general (perturbative ${ }^{\mathrm{b}}$ ) result is the construction of an (effective) Hamiltonian operator for the system with the general form:

$$
\begin{aligned}
\hat{H}_{\text {eff }} & =\hat{H}_{\text {free }}\left(\hat{\Phi}^{\dagger}, \hat{\Phi}\right)+\sum_{\mathbf{m}} \theta^{\mu \nu}(\mathbf{m})\left(\hat{L}_{\mu}\right)^{\dagger}(\mathbf{m}) \hat{L}_{\nu}(\mathbf{m})+ \\
& +\hat{H}_{\text {mixing }}\left(\hat{\Phi}^{\dagger}, \hat{\Phi},\left(\hat{L}_{\mu}\right)^{\dagger}, \hat{L}_{\mu}\right)
\end{aligned}
$$

where $\hat{H}_{\text {free }}$ is the Hamiltonian corresponding to the free massless field theory, the second term is a pure dynamical-diffeomorphism quadratic contribution to the energy $\left(\theta^{\mu \nu}(\mathbf{m})\right.$ is a $c$-number function on $\mathbf{m}$ which closely depends on the inverse of the cocycle $\left.c_{\mu \nu}^{i}(\mathbf{m}, \mathbf{n})\right)$ and $\hat{H}_{\text {mixing }}$ corresponds to higher-power terms involving a potential mixing among all the operators. Appearing perhaps as an odd phenomenon, the lowest-order term producing interaction is not found inside $\hat{H}_{\text {mixing }}$, but already in the quadratic diffeomorphism one, the reason being the non-canonical form of the commutators in (1), in particular the second one. This will be apparent in a specific example below. Regarding the expression (2), our claim is that the terms correcting the free Hamiltonian, could account for the mass terms in the effective theory.

Finally, we are in the position of unambiguosly stating our conjecture: a crucial part of mass generation can be phenomenologically described as a (radiative) correction resulting from the interaction between the massless fields and some effective degrees of freedom appearing from the mere existence of particles.

${ }^{b}$ A crucial step of GAQ consists in exponentiating the starting Lie algebra. When the latter is infinite-dimensional this is a enormous task and only an order-by-order procedure is generally feasible, leading to perturbative though renormalized results. 
Of course, a real prediction of this contribution to the mass would require a knowledge of the underlying fundamental theory, since it plays the role of fixing the values of the extensions in the algebra (11) and therefore of the key $\theta^{\mu \nu}(\mathbf{m})$. Beyond that, the mechanism is not sensitive to the higher-energy detailed description which could find support on strings theory, loop quantum gravity, a non-commutative version of geometry, a more sophisticated QFT or another effective yet more fundamental model, such as worm-holes (see 12 ) in Euclidean quantum gravity. A serious attempt to provide a realistic example in this context deserves a careful analysis on the potential anomalous breaking of diffeomorphism invariance in current candidates for fundamental theories. For the time being, we present an over-simplified illustrative example, consisting of a free real scalar field in one (compact) spatial dimension. With the ansatz that only the spatial diffeomorphisms become dynamical, the relevant algebra is:

$$
\begin{aligned}
{\left[\hat{L}_{m}, \hat{L}_{n}\right] } & =(m-n) \hat{L}_{m+n}+c m^{3} \delta_{m+n, 0} \\
{\left[\hat{L}_{m}, \hat{a}_{n}\right] } & =-n \hat{a}_{m+n} \\
{\left[\hat{a}_{m}, \hat{a}_{n}\right] } & =m \delta_{m+n, 0}
\end{aligned}
$$

The Hilbert space is constructed from a unique vacuum state $\mid 0>$, by applying the creation operators $\hat{\alpha}_{n}^{\dagger} \equiv \frac{1}{\sqrt{n}} \hat{a}_{-n}$ and $\hat{L}_{n}^{\dagger} \equiv \hat{L}_{-n}$, with $n>0$, and where the annihilation operators are given by $\hat{\alpha}_{n} \equiv \frac{1}{\sqrt{n}} \hat{a}_{n}$ and $\hat{L}_{n}(n \geq 0)$. The operators $\hat{\alpha}_{n}^{\dagger}, \hat{\alpha}_{n}$ now satisfy the standard canonical commutators: $\left[\hat{\alpha}_{n}, \hat{\alpha}_{m}^{\dagger}\right]=\delta_{n, m}$. The perturbative calculation of the Hamiltonian of the system (which can be derived from Noether invariants in 13 , together with a proper setting in the values of the central extensions there) yields:

$$
\hat{H}=\sum_{n>0}\left(n \hat{\alpha}_{n}^{\dagger} \hat{\alpha}_{n}+\frac{1}{c n^{2}} \hat{L}_{n}^{\dagger} \hat{L}_{n}+\ldots\right)
$$

The expression from the standard treatment of a free field with mass $M$ presents only a term $\hat{H}_{\text {field }}=\sum_{n>0} \sqrt{M^{2}+n^{2}} \hat{\alpha}_{n}^{\dagger} \hat{\alpha}_{n}$ (we are explicitly omitting zero-energy terms). Taking into account that the expression in (伍) is only perturbative, we must look for a regime in which we can coherently compare it with $\hat{H}_{\text {field }}{ }^{\mathrm{c}}$. This can be achieved by expanding the field dispersion relation for large $n$. Thus we have

$$
\hat{H}_{\text {field }}=\sum_{n>0} n \sqrt{1+\frac{M^{2}}{n^{2}}} \hat{\alpha}_{n}^{\dagger} \hat{\alpha}_{n}=\sum_{n>0}\left(n \hat{\alpha}_{n}^{\dagger} \hat{\alpha}_{n}+\frac{M^{2}}{2 n} \hat{\alpha}_{n}^{\dagger} \hat{\alpha}_{n}+\ldots\right)
$$

The explicit way of comparing both approaches is accomplished by evaluating (里) and (5) on physical states, in particular on 1-particle states $\left|k>=\hat{\alpha}_{k}^{\dagger}\right| 0>$. One can

${ }^{\mathrm{c}}$ The comparison with the corresponding expression in a first-quantization theory such as the standard treatment of the bosonic string would be formally more straightforward, but this is not our aim here. 
raise the question about the convenience of using $\left|k>=\hat{\alpha}_{k}^{\dagger}\right| 0>$ to implement the physical 1-particle states in the theory with dynamical diffeomorphisms, where the excitation of these effective modes would suggest the possibility of a more general linear combination containing $\hat{L}_{k}^{\dagger} \mid 0>$ states. At worst, one could consider (4) in the spirit of perturbation theory over a free massless scalar field, thus using the nonperturbated $\left|k>=\hat{\alpha}_{k}^{\dagger}\right| 0>$ to evaluate first-order corrections to the energy levels. This is the approach we shall use here.

The correction to the excitation energy of the massless particle, calculated in the quantum effective theory derived from (3), accounts for the energy of the interaction with the effective diffeomorphism degrees of freedom. Even though this energy could show a complicated behaviour in the momentum of the particle, we separate the intermediate (low) and very high-momenta dependence and attempt to extract the energy related to the mass out of the form of the interaction energy at the lowest appearing momenta. Therefore, when evaluting the second term in (41) (which we shall denote by $\hat{H}_{L}$ ) we expect to find an expression that can be identified with the one coming from the second term in (5) plus an energy corresponding to very high-momenta dependence $\left(E_{h-m}\right)$ :

$$
\frac{<k\left|\hat{H}_{L}\right| k>}{<k \mid k>}=\frac{M^{2}}{2 k}+E_{h-m}
$$

The evaluation of the first member gives the finite result:

$$
\frac{<k\left|\hat{H}_{L}\right| k>}{<k \mid k>}=\frac{1}{c} \sum_{n=1}^{k-1} \frac{k(k-n)}{n^{2}} .
$$

The identification of the particle-mass out of (7) is guaranteed by the existence of terms in the sum with a $k^{-1}$ behaviour (using large $k$ ). Therefore,

$$
\frac{M^{2}}{2 k} \sim \frac{1}{c k} \Rightarrow M \sim \sqrt{\frac{2}{c}} .
$$

The rest of the energy is in fact a very high-momenta correction to the interacting energy, implying high-energy deviations from the dispersion relation we are trying to match. The inclusion of these corrections in a corresponding field theory without diffeomorphisms, would imply the correction of the propagator of the free field. Therefore, as long as we do not deal with very high energies, a field theory of a Klein-Gordon scalar field with mass $M=\sqrt{\frac{2}{c}}$ is a good model of the effective quantum theory defined by (3), thus involving a rationale for the origin of the mass parameter in the field theory. However, when we extend the application range of the Klein-Gordon model beyond its limits, we are disregarding the above mentioned high-momentum interacting energy and, as a matter of fact, we are decoupling it from the non-gravitational interactions. We have then an energy, and therefore a source for the gravitational field dynamics described by Einstein equations (which 
we decoupled from the very beginning) that is not seen by the rest of physical interactions, and thus it is completely dark. It is tempting to suggest speculatively this as an avenue towards the dark-matter problem. When studying the two-particle states, we find

$$
\begin{aligned}
\frac{<k l\left|\hat{H}_{L}\right| k l>}{<k l \mid k l>} & =\frac{k}{c} \sum_{n=1}^{k-1} \frac{k-n}{n^{2}}+\frac{l}{c} \sum_{n=1}^{l-1} \frac{l-n}{n^{2}}+ \\
& +\frac{k l}{c\left(1+\delta_{n, k}\right)}\left(\frac{1}{(k+l)^{2}}+\frac{\delta_{k \neq l}}{(k-l)^{2}}\right),
\end{aligned}
$$

where the first two terms in r.h.s. correspond to the masses of the particles and its high-momenta corrections while the third term can be interpretated as an extra energy needed to maintain the particles separated (note that it is positive, so that we must do some work to have separated particles).

Finally, we should point out that the use of the Virasoro algebra does not turn the two-dimensional example into a too special case, since a direct generalization to higher dimensions is in fact provided by the non-central Virasoro-like extension 8 obtained by making $c_{\mu \nu}^{i}(\mathbf{m}, \mathbf{n})=c m_{\mu} n_{\nu}\left(m^{i}-n^{i}\right)$ and $\hat{A}_{i}(\mathbf{m})=\hat{S}_{i}(\mathbf{m})$ in (1) $)$, although involving much more cumbersome expressions. The only aim of the presented example is that of providing a taste of and sparking intuition for the potentialities of expressions (2) and (4), the real point we wish to emphasize. Rigorous analyses require a subtle weaving together of the possible extensions in (11), the field dispersion relation we seek to fit and the possibility of more complex settings in which those elements may act.

In conclusion, we have posed a simple framework for mass generation of particles by proposing a mechanism capable of endowing massless free fields with a non-zero mass. Even if this is a tiny effect, it would provide a non-zero germ suitable of being amplified with other mechanisms such as the multiplicative renormalization appearing in QED for the electron. The present description has an effective nature and is quite insensitive to the underlying fundamental theory, provided that diffeomorphism invariance plays a fundamental role. This last point is reinforced by Mach's conceptual intuitions linking inertia and Gravity. A heavy use of the image of particle creation as an inherently quantum process is displayed. This can be explicitly seen in the example discussed by underlining the dependence of particle masses on the central charge of the Virasoro algebra in (3), in such a way that they vanish in the classical limit $c \rightarrow \infty$ (see 14 ), thus revealing themselves as a quantum phenomenon. Let us finally state that, though the presence of (non-gravitational) interactions is crucial in ascribing a point-like nature to the particles in QFT, once we accept such a nature we can get rid of those interactions and deal essentially with free theories as a starting point for the proposed mechanism. Therefore, even if our conjecture of relating a mass origin for particles to the interaction with the some effective degrees of freedom does not fully work, the proposed radiative corrections could imply important and observable consequences on the energy spectrum of free 
fields, in particular entailing modifications in the field propagators.

\section{Acknowledgments}

We want to thank C. Barceló for crucial discussions and his continuous support. We also want to thank A.P. Balachandran for stimulating comments.

\section{References}

1. H.A. Lorentz, Proc. Acad. Sci., Amsterdam 6 (1904). Reprinted in A. Einstein et al., The Principle of Relativity, Dover (1952).

2. C. Bernard et al., Nucl. Phys. Proc. Suppl. 73, 198 (1999).

See also F. Wilczek, Physics Today, Nov. 1999, 11 and F. Wilczek, hep-ph/0201222.

3. L. M. Brown (ed.), Renormalization, Springer-Verlag (1993).

4. A. Pressley and G. Segal, Loop groups, Oxford University Press (1986).

5. L.D. Faddeev and S.L. Shatashvili, Phys. Lett. 167B, 225 (1986).

6. J. Preskill, Ann. Phys.(N.Y.) 210, 323 (1991).

7. A. Dzhumadil'daev, Z. Phys. C 72, 509 (1996).

8. T.A. Larsson, math-ph/0002016.

9. V. Aldaya, J. Navarro-Salas and A. Ramírez, Commun. Math. Phys., 121, 541 (1989).

10. A.A. Kirillov, Elements of the theory of representations, Springer Verlag (1976).

11. N.M.J. Woodhouse, Geometric Quantization, Oxford University Press (1991).

12. S. Coleman, Nucl. Phys. B310, 643 (1988).

13. V. Aldaya and J.L. Jaramillo, Class. Quant. Grav. 17, 1649 (2000).

V. Aldaya and J.L. Jaramillo, Class. Quant. Grav. 17, 4877 (2000).

14. E. Witten, Commun. Math. Phys. 114, 1 (1988). 